Trauma Berufskrankh $2006 \cdot 8$ [Suppl 1]: S85-S86 DOI 10.1007/s10039-005-1093-8

Online publiziert: 26. Januar 2006

(C) Springer Medizin Verlag 2006
Th. Köhler

Landesverband Südwestdeutschland der gewerblichen

Berufsgenossenschaften, Heidelberg

\section{Umsetzung der Freiburger Arbeitsunfall-Studie in die Praxis}

\author{
Diskussion mit dem Auditorium
}

Nachdem bei der Unfallmedizinischen Tagung 2003 des Landesverbands Südwestdeutschland der gewerblichen Berufsgenossenschaften das Modellverfahren der Unfallversicherungsträger zum Thema Arbeitsunfall und psychische Gesundheitsschäden vorgestellt worden war, stand bei der Tagung des Jahres 2005 die „Freiburger Arbeitsunfallstudie“ im Mittelpunkt (zur Zusammenfassung der Diskussion aus der Tagung 2003 vgl. Köhler u. Schlickewei [1]). Auch die Diskussion am 22.10.2005 zeigte ein großes Interesse sowohl der ärztlichen Teilnehmer als auch der Mitarbeiter berufsgenossenschaftlicher Verwaltungen an der Thematik psychischer Störungen nach Unfalltraumen. Aus der umfassenden Diskussion sind folgende Punkte von allgemeinem Interesse:

In der unfallchirurgischen Abteilung der Universitätsklinik Freiburg ist als wichtige Konsequenz aus der Freiburger Arbeitsunfallstudie eine Psychologin eingestellt worden. Diese führt nach den Worten von Herrn Prof. Südkamp das Screening aller Patienten durch, unabhängig vom BG-Status; dadurch ist sichergestellt, dass auch auf psychischem Fachgebiet eine ggf. notwendige Diagnostik und Behandlung erfolgen. Ein gesamtheitlicher Ansatz wird nicht nur bei organischen, sondern gerade auch bei psychischen Erkrankungen als unabdingbar angesehen.

Aus dem Publikum wurde gefragt, warum nur $3 \%$ der in die Studie einge- schlossenen Probanden eine psychotherapeutische Intervention erfahren hätten, obwohl bei etwa 15\% der Patienten psychische Beschwerden in den katamnestischen Untersuchungen festgestellt worden seien. Dr. Angenendt vermutete, dass sowohl Durchgangsärzte als auch UV-Träger bei der Heilverfahrenssteuerung die mögliche psychische Komponente eines Traumas noch nicht ausreichend in Betracht ziehen würden. Prof. Südkamp ergänzte, dass in der Klinik tätige Durchgangsärzte verstärkt auf ihre Patienten einwirken sollten, eine quasi der Chirurgie angegliederte psychotherapeutische Behandlung in Anspruch zu nehmen.

Zum Ablauf der psychotherapeutischen Intervention auf der unfallchirurgischen Station führte Prof. Südkamp aus, dass für den persönlichen, intensiven Dialog zwischen Patient und Psychotherapeut stets ein Einzelzimmer oder ein spezielles Untersuchungszimmer zur Verfügung gestanden habe.

Wichtiger Diskussionspunkt aus dem Publikum war auch die Frage, ob man durch das Screening nicht erst eine Sensibilisierung der Verletzten erreiche und dadurch letztlich die Geltendmachung von psychischen Störungen provoziere. Dr. Angenendt entkräftete diese Befürchtung damit, dass im Gegenteil Schwierigkeiten bestanden hätten, für die Studie genügend Hochrisikopatienten zu rekrutieren. Man habe deswegen auf die BG-Kliniken und auf weitere Kliniken zurückgreifen müssen. Die vorhergesagten erhöhten Inzi- denzen von psychischen Störungen hätten sich definitiv nicht bestätigt.

Thematisiert wurde auch, wie der beste Zeitpunkt für eine psychotherapeutische Intervention bestimmt werden kann. Nach Auffassung von Dr. Angenendt ist es ein Ergebnis der Studie, dass die eingeleiteten intensiveren Maßnahmen bei den Hochrisikopatienten offensichtlich noch in einem zu frühen zeitlichen Korridor gelegen hätten. Die Patienten seien durch das Setting der stationären unfallchirurgischen Behandlung noch zu stark absorbiert. Deshalb seien am Anfang eher eine Verlaufsbeobachtung sowie die Edukation und Aufklärung angezeigt. Die Identifikation der Hochrisikopatienten solle also noch durch die Klinik geleistet werden, während eine intensivere psychotherapeutische Intervention regelmäßig in die ambulante Weiterbehandlung zu verlagern sei. Ausnahmen seien die Fälle, bei denen offensichtlich ein sofortiger Handlungsbedarf besteht und eine entsprechende konsiliarärztliche Hinzuziehung erforderlich ist.

In Fällen mit intensiv-medizinischer Behandlung schlug Frau DrechselSchlund für die Umsetzung der Studienergebnisse vor, das Screening bei diesen Schwerletzten etwa 20 Tage nach der Verlegung von der Intensiv- auf die Normalstation durchzuführen. Letztlich sei mit den Kliniken, die sich an dem geplanten praktischen Erprobungslauf beteiligen wollten, ein entsprechend geeigneter Zeitpunkt abzustimmen. 
Problematisiert wurden in der Diskussion auch die Sprach- und Verständnisprobleme des zunehmenden Anteils ausländischer Patienten. Dr. Angenendt wies darauf hin, dass nach dem Studiendesign deutsche Sprachkenntnisse vorausgesetzt waren und daher aus der Studie keine speziellen Erfahrungen zu diesem Punkt gewonnen werden konnten.

Auf die Umsetzung der Studienergebnisse zielte auch die Frage, wie der weiterbehandelnde niedergelassene Durchgangsarzt erfahre, ob ein Patient als „Behandlungsfall“ oder als „Beobachtungsfall“ eingestuft worden sei. Dr. Angenendt erläuterte, dass in einem zukünftigen Verfahren geplant sei, dass die Klinik sowohl dem Durchgangsarzt als auch dem UV-Träger das Screeningergebnis in geeigneter Weise mitteilt. Dann sei es Aufgabe der BG oder des Durchgangsarztes, eine Konsiliarvorstellung zu initiieren, wenn der Patient einen Screeningwert $>7$ erreicht.

Köhler fasste die Diskussion zusammen und stellte fest, dass nach den Ergebnissen der Freiburger Arbeitsunfallstudie keine „Büchse der Pandora“ geöffnet worden sei. Psychische Störungen nach Arbeitsunfällen seien ein überschaubares Problem, dessen sich die Unfallversicherung nach dem gesetzlichen Rehabilitationsauftrag „mit allen geeigneten Mitteln“ anzunehmen hätte. Eine Heilverfahrenssteuerung sei nur lege artis, wenn auch die Aspekte psychischer Störungen berücksichtigt würden - das gelte sowohl für die Durchgangsärzte als auch für die UV-Träger.

\section{Korrespondierender Autor}

\section{Th. Köhler}

Landesverband Südwestdeutschland der gewerblichen Berufsgenossenschaften

69004 Heidelberg

tkoehler@heidelberg.lvbg.de

\section{Literatur}

1. Köhler Th, Schlickewei W (2004) Arbeitsunfall und psychische Gesundheitsschäden - ein Modellverfahren der Unfallversicherungsträger - Diskussion mit dem Auditorium. Trauma Berufskrankh [Suppl 1] 6: $S 61$ 